\title{
Digital Imaging in Dental Impressions
}

\author{
${ }^{1}$ Mohan Bhuvaneswaran, ${ }^{2}$ Chitra JV Vekaash
}

\begin{abstract}
Digital imprint and computer-aided design/computer-aided manufacturing (CAD/CAM) systems offer a wide range of benefits compared to traditional techniques. The introduction of intraoral scanners has radically changed the way in which the dentists approach the restorative workflow. The constant evolution of the hardware and software in relation to this has made a digital end-to-end restorative solutions a reality. Precision, accuracy and hassle-free procedures have made these systems user-friendly and patient-friendly. There are many advantages in using this advanced technology. This article will provide the information on digital dental impression and clinical application of the same.
\end{abstract}

Keywords: Computer-aided design/computer-aided manufacturing, Dental imaging, Digital dentistry, Digital impressions, Laser scanner, LED scanners, Oral digitizers, Oral scanners.

How to cite this article: Bhuvaneswaran M, Vekaash CJV. Digital Imaging in Dental Impressions. J Oper Dent Endod 2018;3(2):63-70.

\section{Source of support: Nil}

Conflict of interest: None

\section{INTRODUCTION}

An accurate replica of the oral structures is mandatory in our day to day clinical practice. They help in diagnosis, treatment planning and fabrication of restorations. Ever since the necessity of the replica has arisen. There has always been a search towards achieving an exact replica. Various materials have been developed in this regard. Creating a negative replica using various impression materials and forming a positive replica of it has been followed till date. Of course, this technique has its drawbacks in the form of material handling, patient acceptance, and accuracy. The advancement in the field of computer technology and the development of CAD/CAM in the engineering field helped us to develop CAD/CAM for dental use. ${ }^{1}$ The use of CAD/CAM in dentistry came into existence in the early 1970s, since then a lot of research went into digital imaging of dental structures. Digital

\footnotetext{
${ }^{1}$ Director, ${ }^{2}$ Consultant

1,2Department of Conservative and Endodontist, Vignesh Dental Hospital, Chennai, Tamil Nadu, India

Corresponding Author: Mohan Bhuvaneswaran, Director, Department of Conservative and Endodontist, Vignesh Dental Hospital, Chennai, Tamil Nadu, India, e-mail: drmohanb@ yahoo.com
}

intraoral imaging represents an innovative method that enables the dentist to construct a virtual computergenerated copy of the hard and soft tissues with the use of lasers and other optical scanning machines. This technology of three-dimensional (3D) digitization was introduced in the 1980s. A lot of technological advancements have happened which has resulted in the present generation of intraoral scanners. ${ }^{2}$ The digital method of making impressions has great accuracy and will for sure reduce the necessity for traditional impression methods in the near future. Patients feel these are more comfortable and easier compared to older methods. The data is stored in specific software for further fabrication of restorations or positive replica or any other appliance manufacturing. This also allows the retrieval of data at any given point of time for further analysis and followups. This article will discuss the various aspects of digital dental impressions. ${ }^{3,4}$

\section{HISTORY}

In the year 1986, the first system for dental office was introduced, and this was marketed as chairside economical restoration of esthetic ceramics (CEREC) 1 By Sirona Dental Systems, a dental division of Siemens Germany. Mörmann and Brandestini at the University of Zurich developed this system. The CEREC 2 and subsequent CEREC 3 were developed to overcome the drawbacks of the previous system. The current CEREC 3D system was introduced in 2003. CEREC is an abbreviation of Ceramic Reconstruction or Chairside Economical Restoration of Esthetic Ceramics. The next machine to take the lead was developed by Brontes technologies Lexington, Massachusetts, USA, as Lava Chairside oral scanner (COS). The same was acquired by $3 \mathrm{M}$ in the year 2006 and was officially launched in the year 2008. This system and the initial CEREC versions used powder to facilitate the scanning process. The Cadent iTero introduced iTero scanners in the year 2007. ${ }^{5}$ Later in the year 2008 D4D Technologies introduced the E4D Dentist system. The evolution of the machines kept happening until the complete refinement happened. The hardware and the software in the present generations have overcome all the drawbacks that were faced earlier, and digital impression which was just a topic of discussion has now become the reality. The current scanners, three shapes from Trios, Planmecca, Carestream 
and the CEREC AC are the machines of the present. The most popular 3 shape scanner was introduced by TRIOS (Denmark) was into the market since 2011. ${ }^{6,7}$ One step ahead was the introduction of FaceHunter for facial scanning by Zirkonzahn. This is useful for the orthodontists for planning procedures related to facial soft and hard tissues (Figs 1 and 2).

\section{Digital Workflow}

The present-day digital dentistry has grown to the level of offering end to end digital solutions for the dentist. The first step is the acquisition of data. Earlier extra oral scanners were primarily used for this purpose. These extra oral scanners could either scan the impressions or scan the die sectioned model. Extraoral scanners can be hand-held (Bench Scanner) (Figs 3 and 4) or full arch scanners (Fig. 5). They are either optical scanners or laser assisted optical scanners. In either of the case, a convention impression was necessary. ${ }^{8,9}$ The advent of present generation intraoral scanners will now slowly limit the use of extraoral scanners. If there is an in-house milling

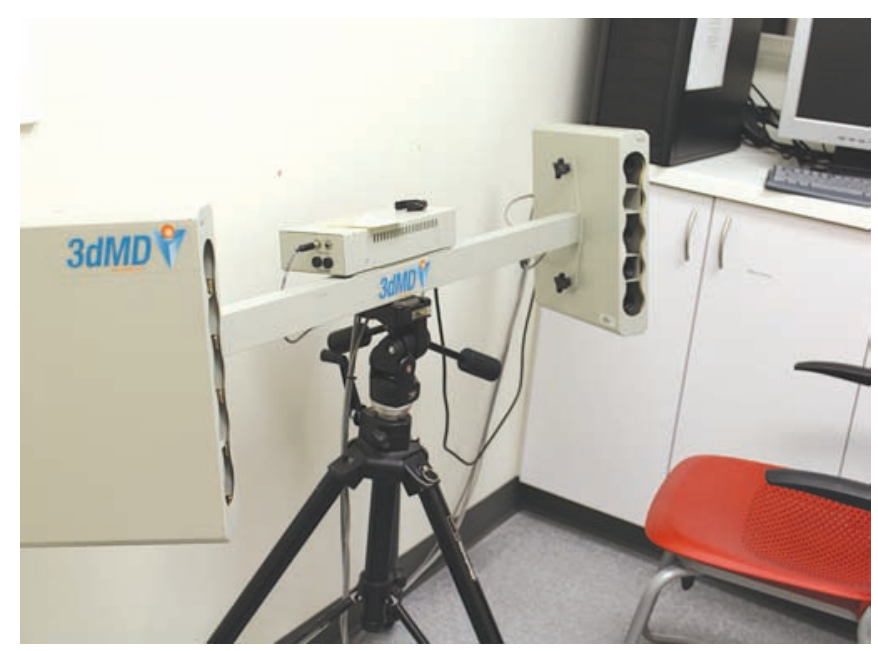

Fig. 1: Face Hunter

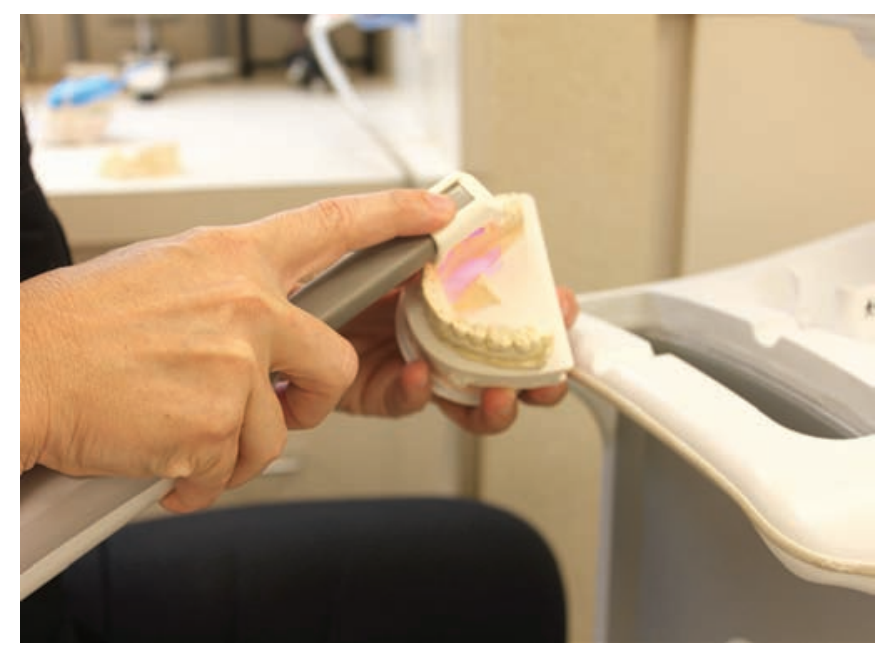

Fig, 3: Hand-held model scanner machine (Figs 6 and 7) the dentist could fabricate restoration by themselves..$^{10}$ Otherwise, a laboratory integration is essential. The acquired data is then sent to the laboratory for either manufacturing of CAD CAM restorations (Figs 8 to 11) or customized restorations with the help of SLA models-3D PRINTING-(Fig. 12). The digital workflow is given in Flow Chart 1.

\section{MECHANISM OF IMAGE ACQUISITION}

The currently available oral scanners use different methods to acquire images. They use different lasers and LED's for imaging. The dental laser technology is based on specific wavelengths. There are particular wavelengths used for hard and soft tissues. The wavelength of diode lasers, which produce infrared waves ranges from 805 to $1064 \mathrm{~nm} .{ }^{11}$ The earlier versions of scanner required a surface coating on the intraoral tissue surface before digital scanning. The Bluecam from CEREC and the COS from $3 \mathrm{M}$ required this powder coating, the entire area that needs to scan has to be coated with a layer of biocompatible Titanium dioxide powder. ${ }^{12}$

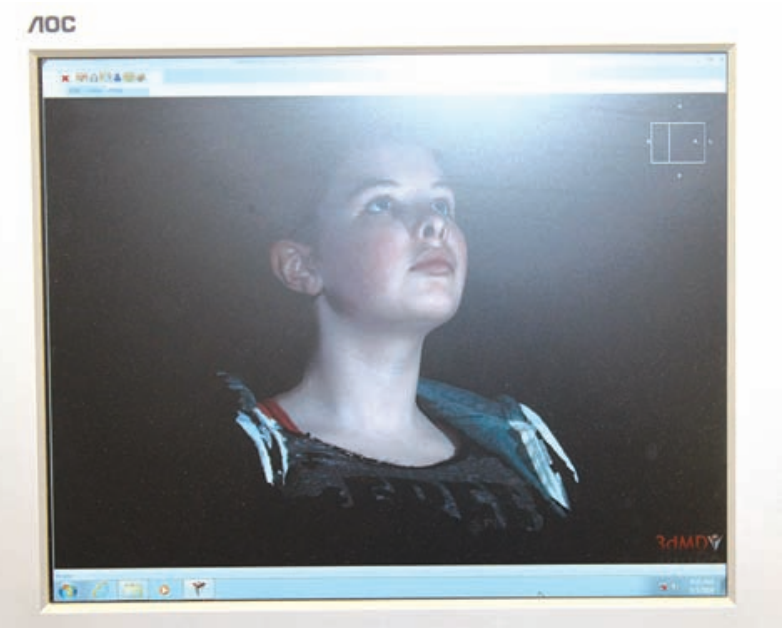

Fig. 2: Image acquired by the Face Hunter

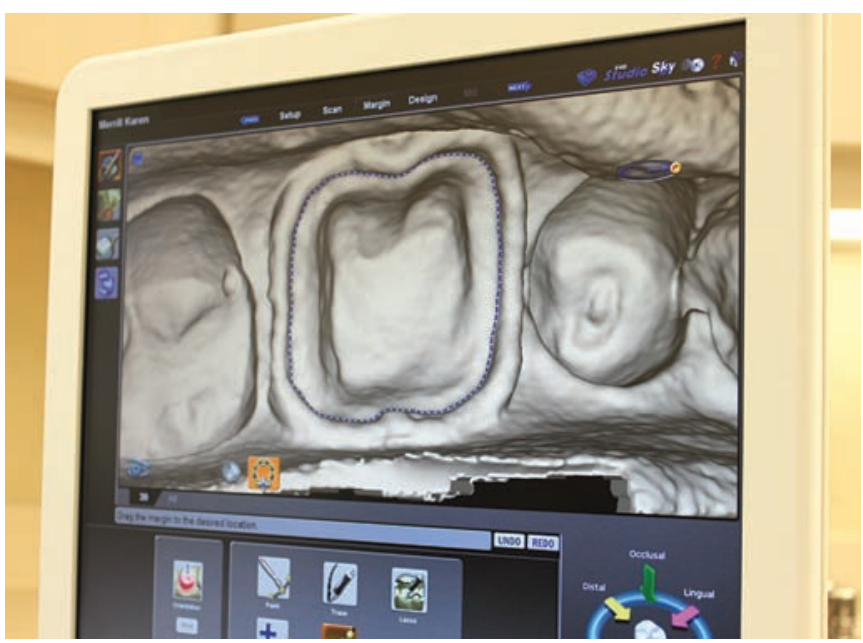

Fig. 4: Image acquired by the model scanner 
This powder can then be easily removed with air and water. But this was a major clinical hinderance, and that is why the systems never became user-friendly, as the clinicians look in for hassle-free technology. That lead to the development of the current generation of powderfree oral scanners.

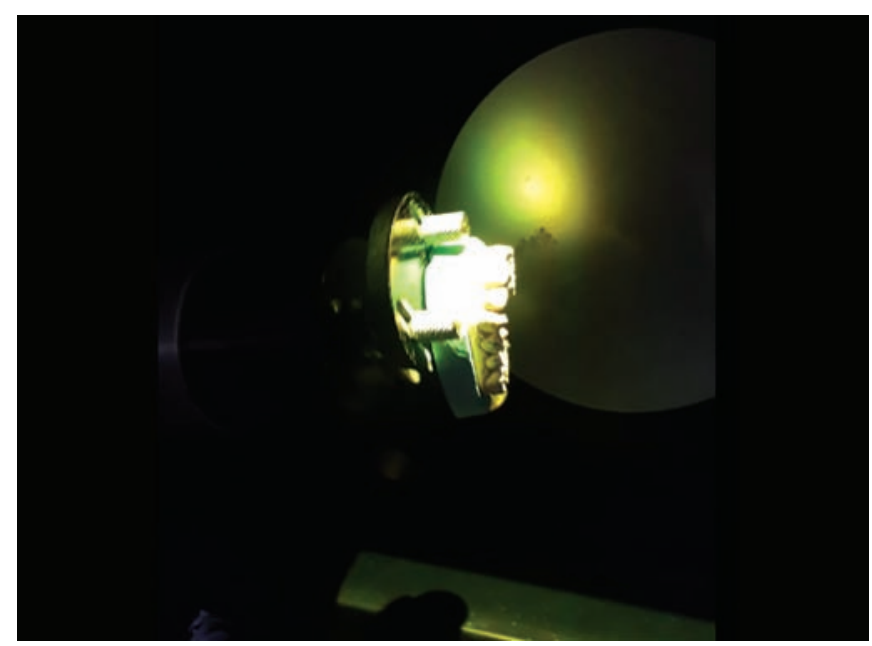

Fig. 5: Full model scanner

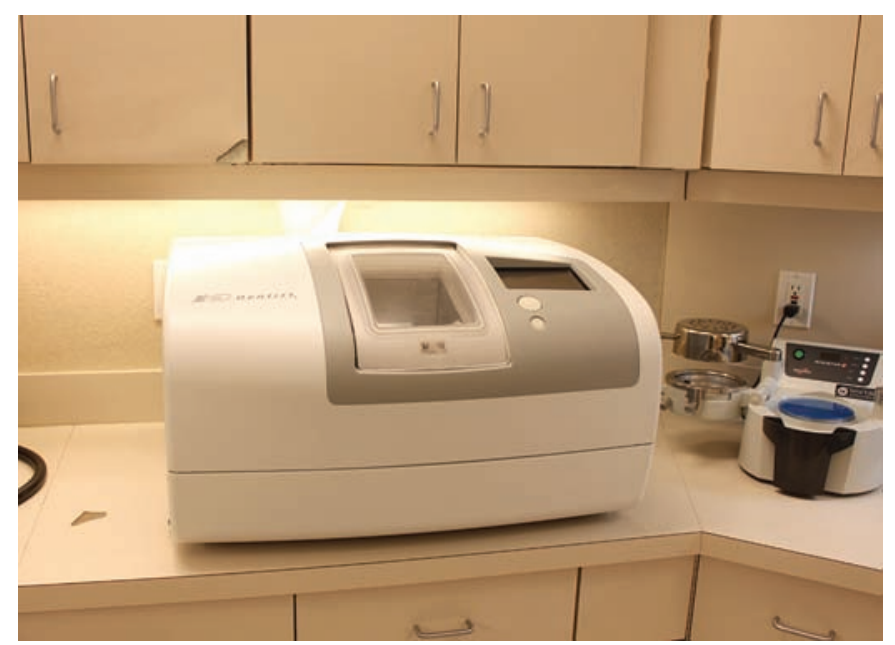

Fig. 7: E4D Milling machine for dental office

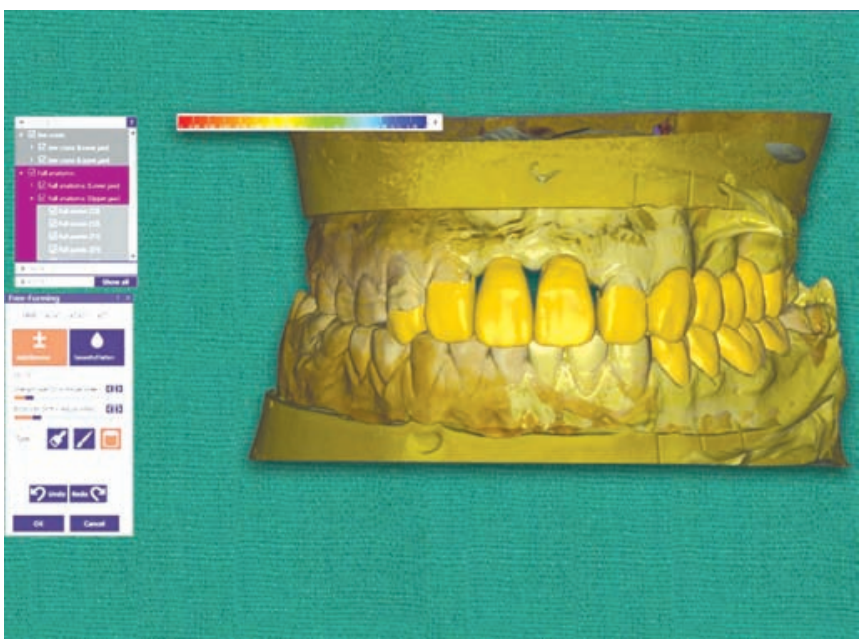

Fig. 9: Digitally designed restoration
Initially, CEREC used longer wavelength infra-red laser light source, but later it was shifted to shorter wavelength visible laser light source. Blue LED that is relevant for $3 \mathrm{D}$ acquisition was used in the scanner, that allowed a greater precision of the resultant optical image. The camera projects a changing pattern of blue

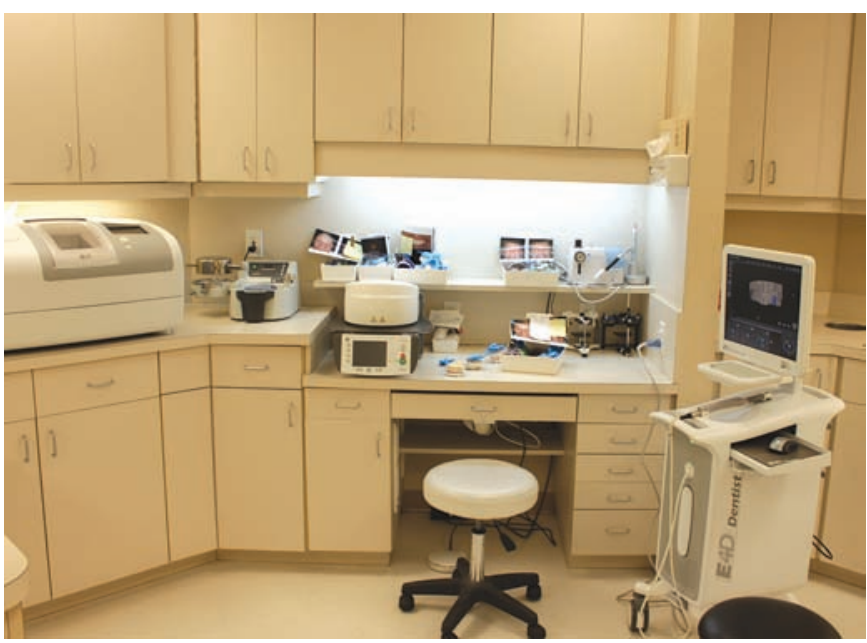

Fig. 6: In-house lab set up

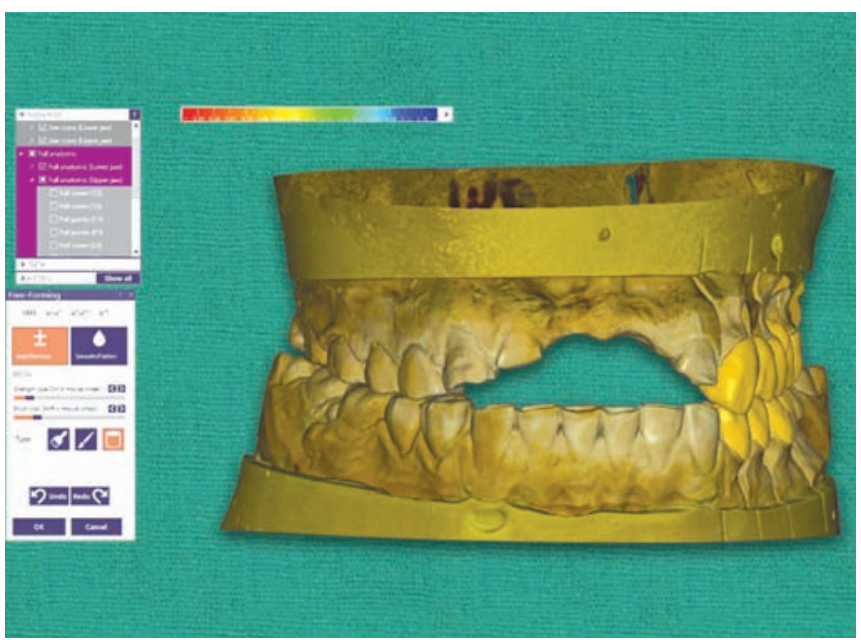

Fig. 8: Digital imaging for designing the restoration

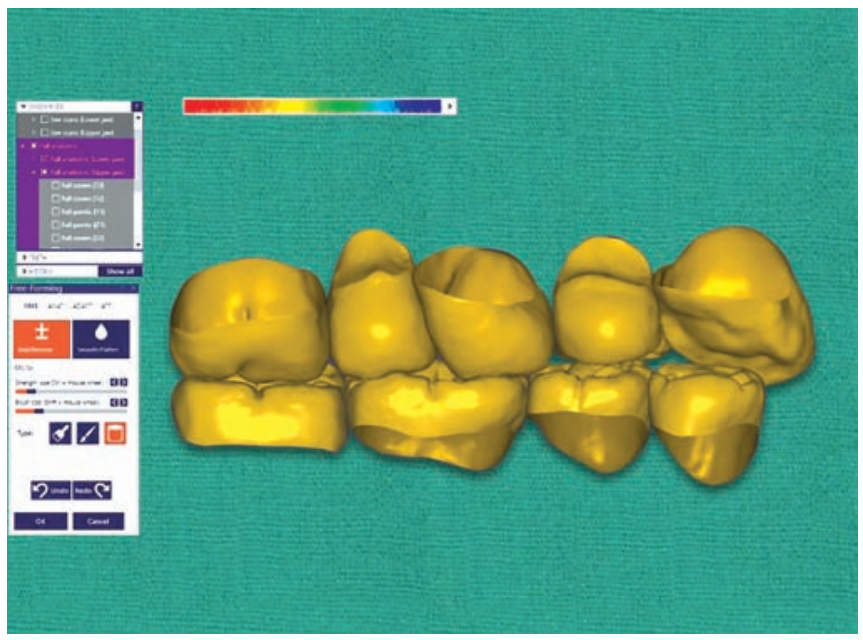

Fig. 10: Image of the resonation 


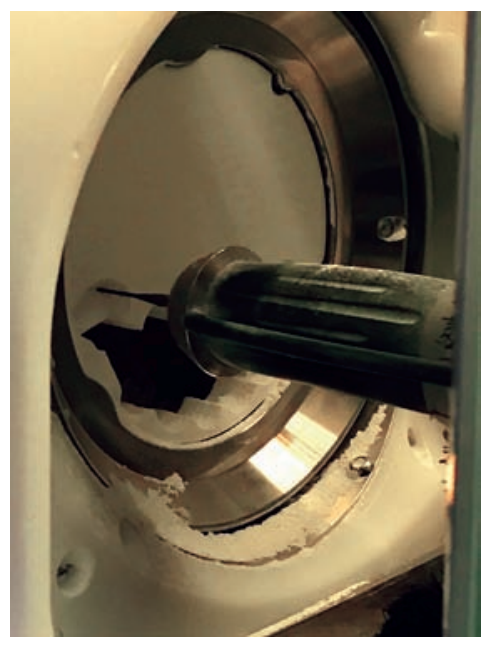

Fig. 11: Milling of the restoration

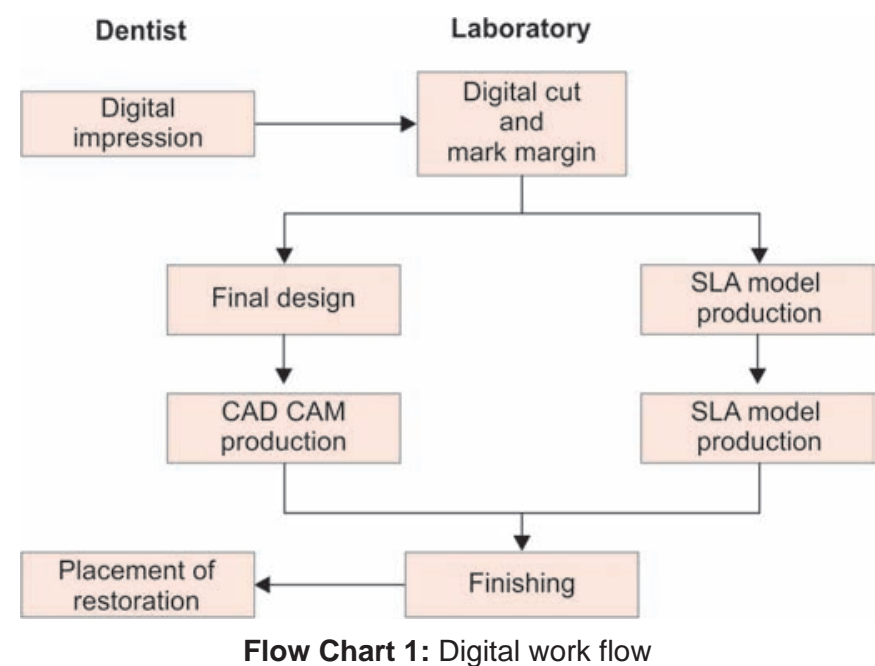

light which is read back in a different angle, and this is called the active triangulation technique. ${ }^{13}$ The telecentric beam allowso capturing of all the details in a single view. The LAVA COS also uses the blue LED to capture the image. But here it is a continuous blue light LED which does the video streaming. This scanner comes along with the CPU in the cart form. The scanner tip contains a complex optical system with multiple lenses. The wand contains almost 192 blue LED cells. However, the accuracy of the powder-free scans is much greater now, and hence these systems that use the powder coating are becoming unpopular.

The CEREC later shifted from Bluecam to the present Omnicam which also uses the blue LED, but it does not require the powder coating. The Omnicom and the CEREC AC Bluecam are the presently available camera from CEREC. The E4D powder free scanner uses the red LED to acquire the data. The iTero system uses the parallel confocal imaging to quickly capture the images, this uses Lasers and optical scanning to digitally capture the oral structures. The camera could focus 300 focal depths and thereby achieves a high-resolution image, this system also does not require powder. ${ }^{14}$

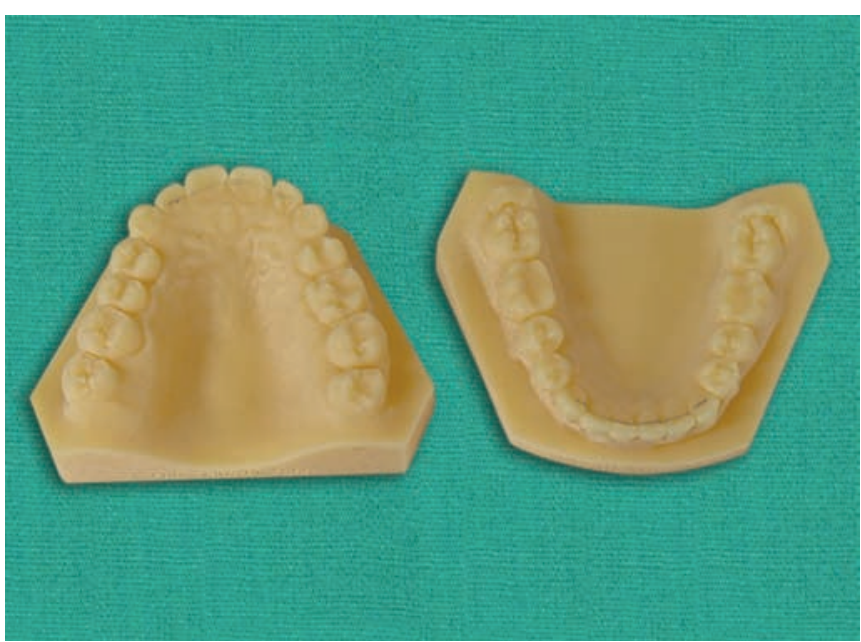

Fig. 12: SLA 3D printed model

The most popular three shape works under the principle of ultrafast optical sectioning and confocal microscopy. The scanning speed is almost 3000 images per second. Thereby the relative movement interference is reduced. ${ }^{15}$ It maintains a fixed spatial relation of the scanner and the object, and hence various focal planes are perfectly recognized. The PlanScan from Planmeca also uses the blue light for capturing the images, The CS 3600 from Carestream uses the triangulation principle in high definition 3D color.

Procuring occlusal records are also essential to get the upper and lower jaw orientation. Each unit has its method of determining the centric. It easy to record the maximum intercuspation using this device, But to guide the patient to centric and then register the centric, a wax is always better.

Following the acquisition, the image is then stored in the particular software of the respective company. The CPU requirements are very special for the scanners. The regular graphics card and the RAM are insufficient to handle the scanners. The Alienware from DELL with an 18 GB ram and Ge-force graphics card are the best suitable ones for the scanners. They can then be then used for internal chair side restoration fabrication, or the images can be sent to a lab for further processing, the images can be used by any hardware-the milling machine, then they are called as open software images, and the system becomes an open system. If the images captured can be used only in specific milling systems then they become closed software images, and the system is called a closed system. It was always wiser to procure an open system scanner so that we are free to manufacture what we require. The software upgrade keeps happening on a regular basis, and we have to make sure that our system gets these upgradations. The files that are sent to the lab are STL files. ${ }^{16,17}$ The lab can from here either print a Stereolithography physical acrylic model for traditional 
manufacturing of restoration or directly complete the entire process in the digital workflow.

\section{CLASSIFICATION}

The scanners are generally classified on the basis of various parameters as given in Flow Chart 2.

\section{Overview of the Systems}

\section{Chairside economical restoration of esthetic ceramics system (Sirona, Bensheim, Germany)}

Cchairside economical restoration of esthetic ceramics (CEREC) 1 system came to market in 1987(first intraoral digital impression with CAD/CAM device). Works on the principle of Triangulation, which uses three straight beams of light intersecting on a focused point. The light source used in CEREC AC Bluecam is blue light and takes a minute to capture one quadrant. Then CEREC AC Omnicam came to the market with the various continuous image capturing technology, where the Bluecam

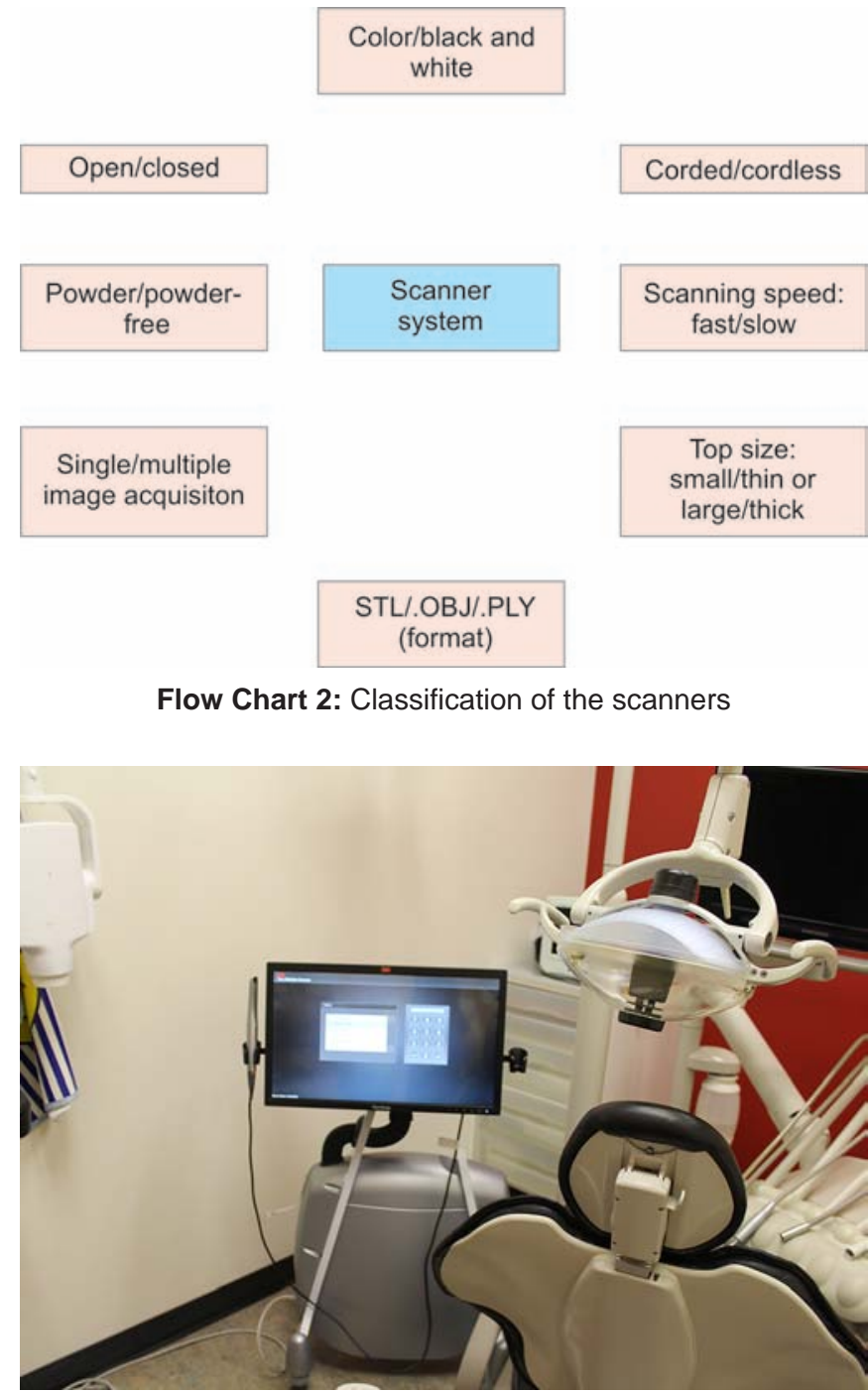

Fig. 13: 3M COS had only single image acquisition, can be used just for a single tooth while Omnicam can be used for a single tooth, quadrant and full arch images. Omnicam also has other advantages like it captures three-dimensional images with prominent natural color. CEREC is a closed system and supports devices like CEREC MC and CEREC In-lab. ${ }^{18}$ Lava C.O.S System (3M ESPE, Seefeld, Germany)

It was invented in 2006 and came to market in 2008. It works on the principle of active wavefront sampling to acquire 3-dimensional data that can also be viewed two-dimensionally. ${ }^{19}$ The scanner uses three sensors at various angles with 192 LEDs and 22 lens which captures images both in focus and out of focus areas using image-processing algorithms. The light source used is visible blue light and scanner tip is $13.2 \mathrm{~mm}$ wide. They work with a mobile host computer with touchscreen display. The models can be virtually altered, corrected and sent to the lab for fabrication of the prosthesis. ${ }^{20}$ (Figs 13 and 14).

\section{iTero system (Cadent Inc, Carlstadt, NJ)}

This system was introduced to the market in 2007. They work on the principle of confocal imaging technique, and the captured images are contoured by laser and visual scanning. The scanning does not require any powder. At about 300 focal depth of tooth structure, 100,000 laser light points fall on the tooth with 50-micrometer separation. + This system captures 3.5 million data points for each arch, making the output of the tooth more precise and accurate. The light source used in this system is a red laser, and the system consists of a computer, mouse, keyboard, and screen with a scanner. ${ }^{21}$ iTero is an open system, and digital files are sent as STL format to any other systems. The scanner takes about 15 to 20 seconds to scan facial, lingual, mesioproximal and distoproximal for each preparation ${ }^{22}$ (Figs 15 and 16).

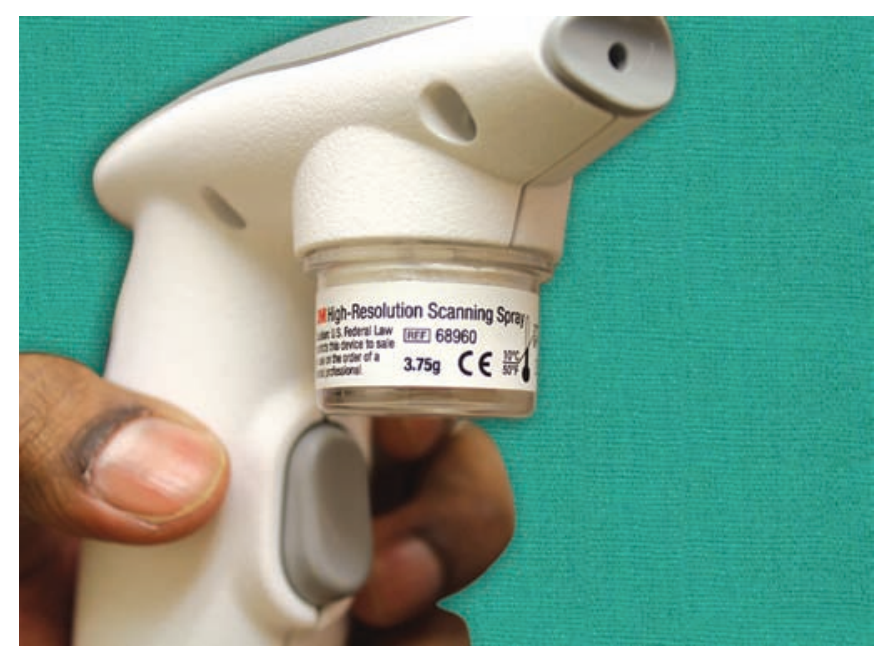

Fig. 14: Powder coating device of 3M 


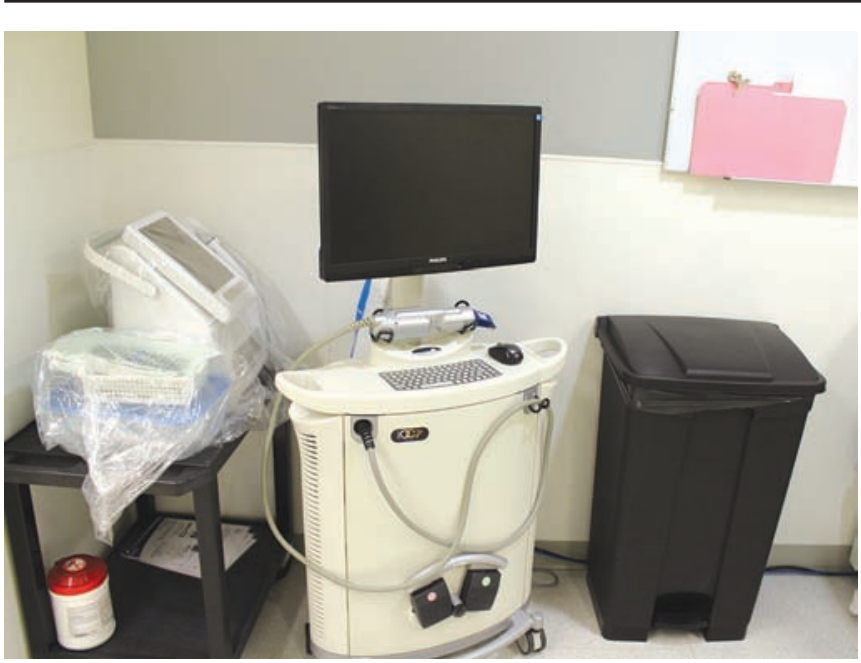

Fig. 15: iTero cart

\section{E4D System}

Was developed by D4D Technologies, LLC (Richardson, TX) and works on the principle of confocal microscopy and optical coherence tomography. They have separate scanning and milling unit in the cart with interunit communication. The light source used is a red laser (high speed) which oscillates at 20,000 cycles per second that stitches continuous images that are captured to construct a 3D model using the software. ${ }^{23}$ Using the rubber stops provided in the scanner, the scanner should be held in a specific distance above the object of interest to be scanned. This scanner does not require any powder to capture the image. The scanned images are transferred to the milling machine using wired or wireless port. ${ }^{24}$

\section{Trios System (3 shape, Denmark)}

This system was introduced in 2010 and reached the market in 2011. This works on the principle of ultrafast optical sectioning and confocal microscopy with powder-free scanning and gives output in the form of 3-dimensional color images of both the teeth and gingiva. Black and white (Trios 3 Mono) version has been introduced which is more economical compared to the colored one. This system is available in both cart and USB version. ${ }^{25}$ The manufacturer provides two different grip design for operator comfort. It is an open system, and the files can be exported to other systems too. This system scans up to 3000 images per second thus reducing the distortion of the scanner probe and object of interest to be scanned ${ }^{26}$ (Figs 17 to 20).

\section{Planscan (Planmeca, USA)}

This scanner is a powder-free system which uses blue laser technology. The scanner is supplied with a USB port that can be connected to laptops. Offers an open system where files can be exported to other systems for

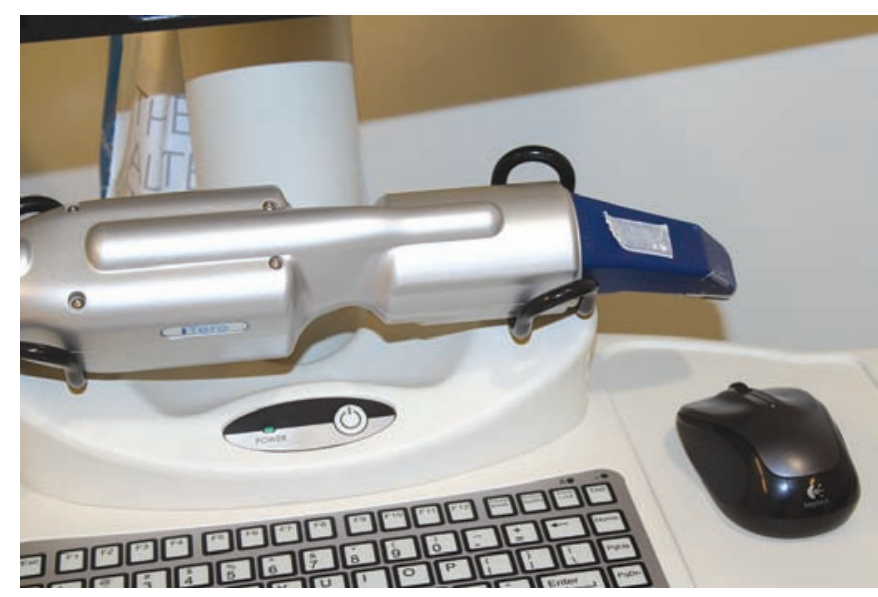

Fig. 16: iTero scanner

milling. The system also provides software to design and a milling device. ${ }^{27}$

\section{CS 3600 (Carestream, United States)}

This scanner works on the principle of triangulation with high definition 3D color. The device is a powderfree system and also an open system available in USB version, where the data can be sent to any other milling device $^{28,29}$ (Fig. 21).

\section{Advantages}

- The accuracy of impressions made.

- Elimination of dirty cabinet and patient discomfort.

- No incorporation of air bubbles.

- No need of storage space or impression materials or cast.

- Patient satisfaction and comfort.

- Time-saving.

- Cross-infection control.

- Easy to adjust and modify the impression.

- Few systems have color scanning and shade selection facilities.

- Easy communication with the lab.

- Retrieval of images at any given point of time.

\section{Disadvantages}

- Expensive

- The difficult learning curve for some individuals.

- Difficult to scan posteriors in patients with restricted mouth opening.

- Inability to capture subgingival margin-Scanners cannot capture what eyes cannot see.

- Less accuracy in capturing images of edentulous arches.

- Closed systems have restricted options in transferring files. 


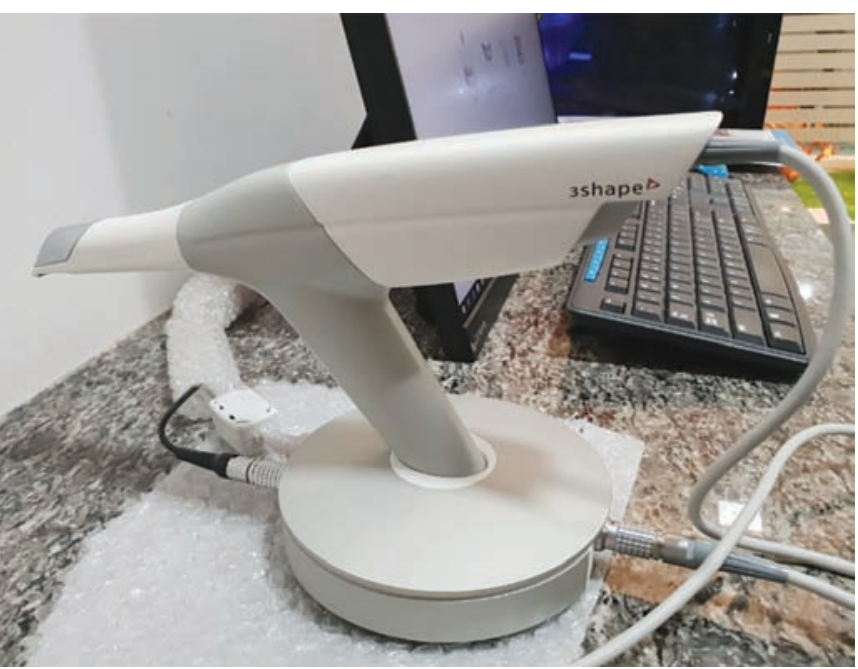

Fig. 17: 3 Shape Scanner

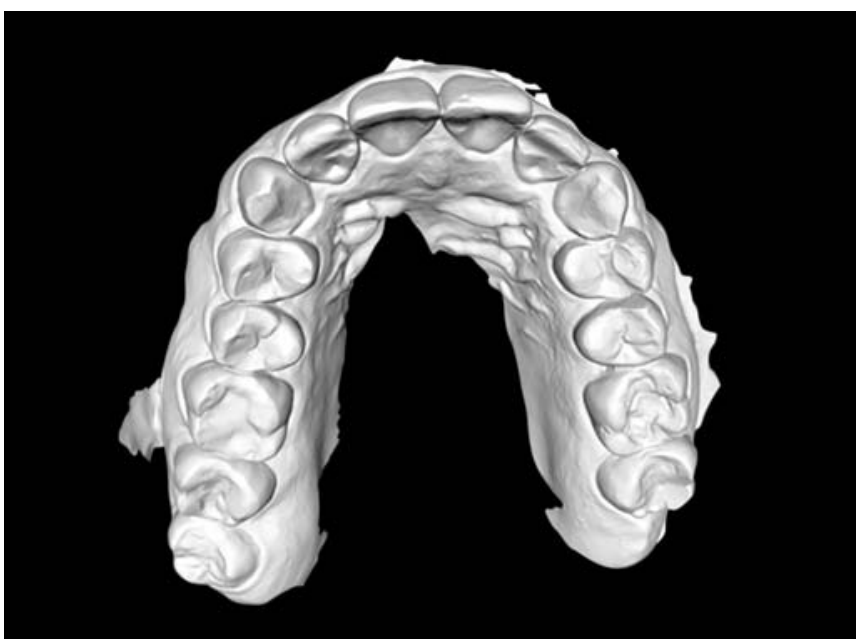

Fig. 19: Digital impression acquired

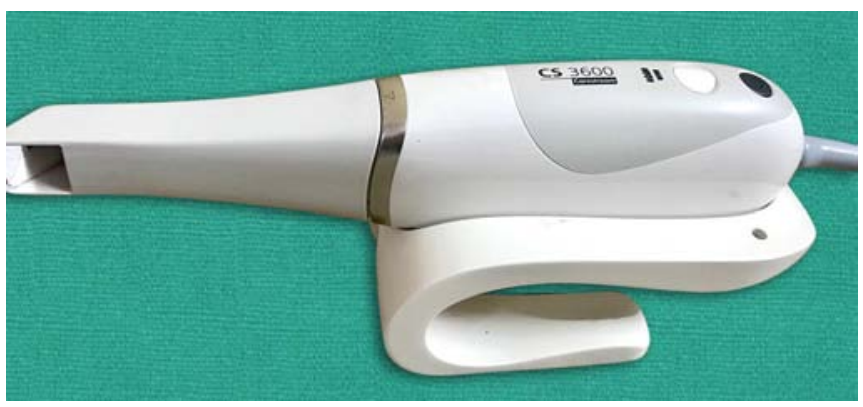

Fig. 21: CS 3600 scanner from Carestream

- Less accuracy of preparation with artifacts due to saliva and blood.

\section{Future}

Digital devices are here to stay and only increase in use in the near future. The growing technology with regard to hard and software will definitely make these equipments affordable. The impression wand and the units will get smaller and more user-friendly. ${ }^{30,31}$ The rate of scanning and producing an accurate image will make the machines more successful. An open platform interface may be

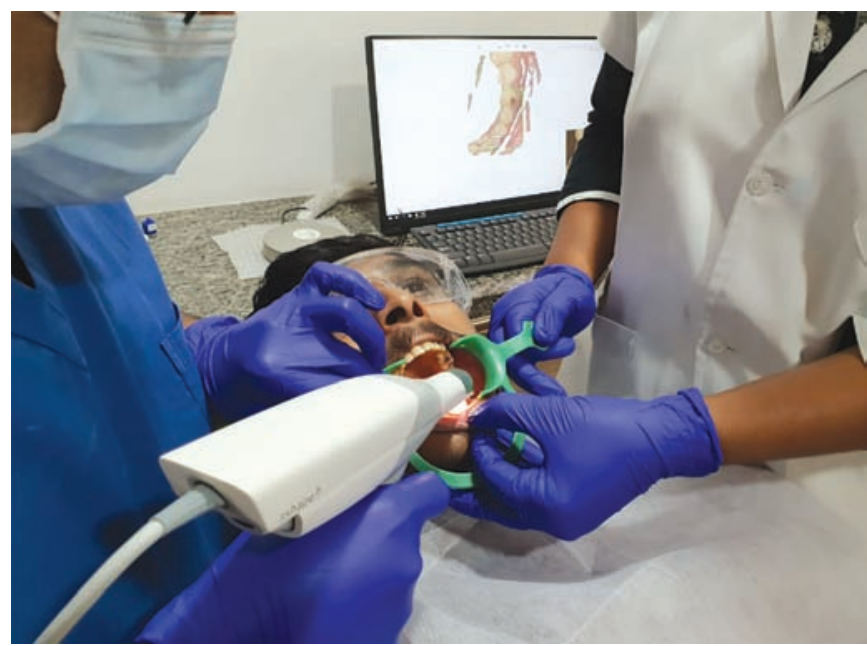

Fig. 18: Acquisition of image

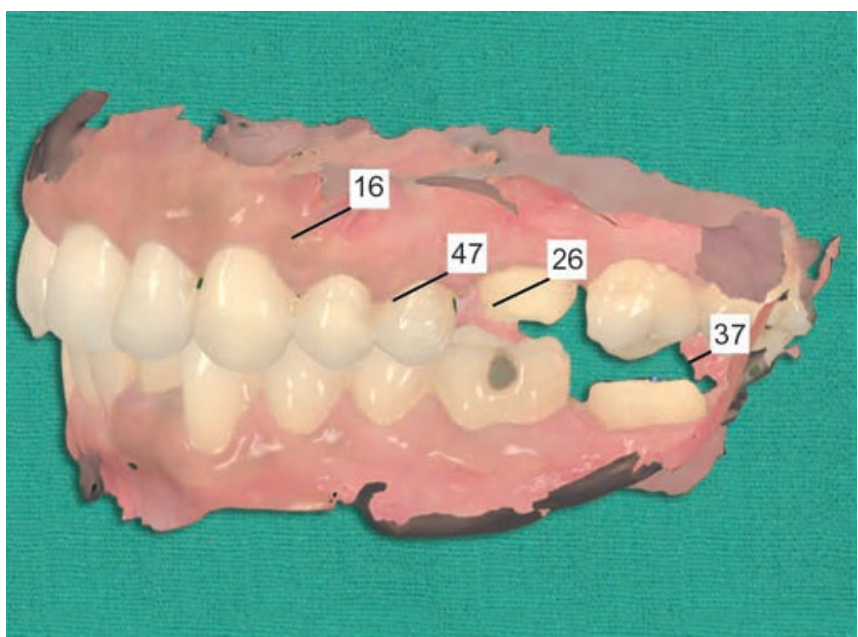

Fig. 20: Digital impression acquired

developed to export and integrate with other imaging modalities like cone beam computed tomography (CBCT) and facial scanners. These files can then be combined and used for diagnosis, treatment planning, and 3D printing to create life-sized analog. The applications of these technologies are voluminous and for sure occupy our majority of day to day clinical practice. Machines that acquire data may be developed with other optical hand-sonic systems. Scanning devices of the future should be able to differentiate between hard and soft tissues and blood and saliva and be able to take rapid and accurate images. ${ }^{32,33}$

\section{CONCLUSION}

Digital dentistry is changing the way in which clinicians can provide indirect restorations which are highly precise restorations and accurate models. This increases the chair side productivity with improved lab communication. All materials and technique developed in dentistry always aim to reduce the technique sensitivity and improve patient comfort with predictable accuracy. Intraoral digital scanners have come a long way, the clinician 
acceptance and usage now become essential to make this digital workflow a success. With a lot of advantages, there are also quite a few drawbacks as discussed in this article. It is also vivid that traditional impressions will stay for certain indications. For digital impressions to become a routine procedure the purchase of the equipment and the initial learning curve are the primary requisites. But for sure the future is digital dentistry.

\section{REFERENCES}

1. Beuer F, Schweiger J, Edelhoff D. Digital dentistry: an overview of recent developments for CAD/CAM generated restorations. Br Dent J. 2008;204(9):505-511.

2. Giordano R, Materials for chairside CAD/CAM-produced restorations. J Am Dent Assoc. 2006;137(suppl):14S-21S.

3. Otto $T$, Schneider D. Long-term clinical results of chairside CEREG CAD/CAM inlays and onlays: A case series. Int J Prosthodont. 2008;21(1):53-59.

4. Sjogren G, Molin M, Van Dijken JW. A 10-year old prospective evaluation of CAD/CAM-manufactured (CEREG) ceramic inlays cemented with chemically cured or dual-cured resin composite. Int J Prosthodont. 2004;17(2):241-246.

5. Posselt A, Kerschbaum T. Longevity of 2328 chairside CEREG inlays and onlays. Int J Comput Dent. 2003;6(3):231-248.

6. Calamia JR. Advances in computer-aided design and computer-aided manufacture technology. CurrOpinCosmet Dent. 1994:67-73.

7. Birnbaum NS, Aaronson HB. Dental impressions using 3D digital scanners: virtual becomes reality. Compend Contin Educ Dent 2008;29(8):494-505.

8. Galhano GA, Pellizer EP, Mazaro JV. Optical impression systems for CAD-CAM restorations. J Craniofac Surg 2012; 23(6):e575-e579.

9. Rekov ED. Dental CAD/CAM systems : a 20-year success story. J Am Dent Assoc 2006;137(Suppl):5S-6S.

10. Mormann WH. The evolution of the CEREC system. J Am Dent Assoc 2006;137:7S-13S.

11. Birnbaum NS, Aaronson HB, Stevens C, Cohen B. 3D digital scanners: a high-tech approach to more accurate dental impressions. Inside Dent 2009;5(4):70-74.

12. Rohaly J. Three-channel camera systems with non-collinearapertures. United States Patent 2006.

13. Kachalia PR, Geissberger MJ. Dentistry a la carte: in-office CAD/CAM technology. J Calif Dent Assoc 2010;38(5)::323-330.

14. Tsitrou EA, Helvatjoglu-Antoniades M, van Noort R: A preliminary evaluation of the structural integrity and fracture mode of minimally prepared resin bonded CAD/CAM crowns. J Dent 2010; 38:16-22.

15. Logozzo S, Franceschini G, Kilpelä A, Caponi M, Governi L, Blois L. A comparative analysis of intraoral 3D digital scanners for restorative dentistry. Int J Med Tech;2011;5(1):1-2.
16. Persson AS, Odén A, Andersson M, Sandborgh-Englund G. Digitization of simulated clinical dental impressions: virtual three-dimensional analysis of exactness. Dent Master 2009;25(7):929-936.

17. Quass S, Rudolph H, Luthardt RG. Difrect mechanical data acquisition of dental impressions for the manufacturing of CAD/CAM restorations. J Dent 2007;;35(12):308-309.

18. Da Costa JB, Pelogia F, Hagedorn B, Ferracane JL. Evaluation of different methods of optical impression making on the marginal gap of onlay created with CEREC 3D. Oper Dent 2010;35(3):324-329.

20. Christensen GJ. Will digital impressions eliminate the current problems with conventional impressions? J Am Dent Assoc 2008;139:761-763.

21. Shembesh M, Ali A, Finkelman M, Weber HP, Zandparsa R An in vitro comparison of the marginal adaptation accuracy of CAD/CAM restorations using different impres- sion systems. J Prosthodont. 2017;26,(7):581-586.

22. Flügge TV, Schlager S, Nelson K, Nahles S, Metzger MC. Precison of intraoral digital dental impressions with iTero and extraoral digitization with iTero and a model scanner. Am J Orthod Dentofacial Orthop. 2013;144(3):471-478.

23. Ahrberg D, Lauer HC, Ahrberg M, Weigl P. Evaluation of fit and efficiency of CAD/CAM fabricated all-ceramic restorations based on direct and indirect digitalization: a doubleblinded, randomized clinical trial. Clinical oral investigations. 2016 Mar 1;20(2):291-300.

24. Fabsinder DJ. CAD/CAM. Inside Dentistry. 2011;7(7):82.

25. Puri S. Evolution in CAD/CAM hardware, software and materials. Dent Today. 2011;30(5):116-121.

26. Masri R, Driscoll C. Clinical applications of digital dental technology. Hoboken (NJ): John Wiley; 2015.

27. Patzelt SB, Lamprinos C, Stampf S, Att W. The time efficiency of intraoral scanners: an in vitro comparative study. J Am Dent Assoc 2014;145(6):542-551.

28. Lee SJ, MacArthur RX, Gallucci GO. An evaluation of student and clinician perception of digital and conventional implant impressions. J Prosthet Dent 2015;110(5):420-423.

29. Cho SH, Schaefer O, Thompson GA, Guentsch A. Comparison of accuracy and reproducibility of casts made by digital and conventional methods. J Prosthet Dent 2015;113(4):310-315.

30. Ender A, Attin T, Mehl A. In vivo precision of conventional and digital methods of obtaining a complete-arch dental impression. J Prosthet Dent 20016;115(3):313-320.

31. Chochlidakis KM, Papaspyridakos P, Geminiani A, et al. Digital versus conventional impressions for fixed prosthodontics: a systemic review and meta-analysis. J Prosthet Dent 2016;116(2):184-190.

32. Zimmermann M, Mehl A, Mörmann W, et al. Intraoral scanning system-a current overview. Int J Comput Dent 2014;18(2):101-129.

33. Miyazaki T, Hotta Y, Kunii J, Kuriyama S, Tamaki Y. A review of dental CAD/CAM: current status and future perspectives from 20 years of experience. Dent Mater J 2009;28(1):44-56. 